\title{
Corpus
}

$14 \mid 2015$

Constitution et usage de corpus en linguistique berbère

\section{Constitution et usage de corpus en linguistique berbère : introduction}

Sabrina Bendjaballah and Samir Ben Si Saïd

\section{(2) OpenEdition}

Journals

\section{Electronic version}

URL: http://journals.openedition.org/corpus/2589

DOI: 10.4000/corpus.2589

ISSN: $1765-3126$

\section{Publisher}

Bases; corpus et langage - UMR 6039

Printed version

Date of publication: 1 January 2015

ISBN: 1 638-9808

ISSN: 1638-9808

\section{Electronic reference}

Sabrina Bendjaballah and Samir Ben Si Saïd, « Constitution et usage de corpus en linguistique berbère :

introduction », Corpus [Online], 14 | 2015, Online since 28 August 2017, connection on 24 September 2020. URL : http://journals.openedition.org/corpus/2589; DOI : https://doi.org/10.4000/corpus.2589

This text was automatically generated on 24 September 2020.

(c) Tous droits réservés 


\title{
Constitution et usage de corpus en linguistique berbère : introduction
}

\author{
Sabrina Bendjaballah and Samir Ben Si Saïd
}

1 Ce numéro de la revue CORPUS est consacré au domaine berbère, l'une des branches de la famille afro-asiatique, et en particulier à la constitution et aux usages de corpus en linguistique berbère. Certains aspects de la structure grammaticale des langues berbères ainsi que de leur diversité étant encore peu connus, l'objectif général de ce volume est de faire progresser notre connaissance de la (micro)variation dans la famille berbère.

2 Tout d'abord, il importe de garder à l'esprit que les langues berbères constituent une famille de langues. L'organisation de cette famille étant sujette à débats, nous suivons Kossmann (2012) et présentons ci-dessous la liste des langues berbères les plus connues, en fonction du pays dans lequel elles sont parlées :

\begin{tabular}{|l|l|}
\hline MAURITANIE & sud ouest $:$ zenaga \\
\hline MAROC & sud ouest : tashelhiyt \\
\hline & centre et sud est : tamazight \\
\hline & nord : tarifit \\
\hline & nord est : Beni Iznasen \\
\hline & Sahara septentrional : Figuig \\
\hline ALGERIE & nord ouest : Beni Snous, Chenoua \\
\hline & nord est : Kabyle, Chaouia \\
\hline
\end{tabular}




\begin{tabular}{|l|l|}
\hline & Sahara septentrional : Ouargla, Mzab, Gourara, Touat (éteint) \\
\hline TUNISIE & Djerba \\
\hline LIBYE & nord ouest : Djebel Nefusa \\
\hline & Sahara : Ghadames, Awjila, Elfoqaha (éteint ?), Sokna (éteint ?) \\
\hline EGYPTE & Sahara : Siwa \\
\hline TOUAREG & $\begin{array}{l}\text { Algérie, Libye : Ahaggar; Niger : Ayer, Iwellemmeden ; Mali : Adagh des Ifoghas ; } \\
\text { Burkina Faso : Oudalan }\end{array}$ \\
\hline $\begin{array}{l}\text { ILES } \\
\text { CANARIES }\end{array}$ & Guanche (éteint au XVII siècle) \\
\hline
\end{tabular}

D’après Kossmann (2012: 18)

3 Si certains sous-groupes de la famille berbère sont bien connus (taqbaylit, tachelhit par exemple), d'autres restent aujourd'hui encore largement sous-étudiés (awjila, guanche par exemple). L'un des objectifs de ce volume est donc d'apporter de nouvelles informations sur ces langues. Ainsi, les contributions de van Putten \& Souag sur le berbère awjila, de Farrujia de la Rosa sur les inscriptions lybico-berbères des îles Canaries et de Kossmann sur le berbère de l'oasis de Figuig, apportent-elles des données et analyses peu connues, de nature à faire significativement avancer notre connaissance de ces langues et au-delà de la famille berbère.

4 Tout travail de terrain précis révèle que les différences entre les langues berbères ne se réduisent pas à des variations dialectales minimes ou insignifiantes. Il s'agit donc tout d'abord de documenter cette variation. Les sources sont d'une part la littérature et d'autre part l'élicitation de données linguistiques. Celle-ci se fait de diverses manières: enquêtes de terrain, travail avec locuteur(s) natif(s) et constitution de corpus. Les articles rassemblés dans ce volume illustrent cette diversité : corpus émergents sur les réseaux sociaux (van Putten \& Souag), corpus oraux et écrits en comparaison (Kossmann), inscriptions lapidaires (Farrujia de la Rosa), ou bien données élicitées auprès de locuteurs natifs dans un réseau structuré de points d'enquête (par exemple Lafkioui et Bendjaballah, Ben Si Saïd \& Haiden).

5 La description linguistique cependant, ne fait sens que si elle est éclairée par la théorie : recueil de données et questionnements théoriques doivent être menés de pair et l'objectif premier reste la validation d'hypothèses ou de prédictions scientifiques. L'objectif est donc de mettre au jour les paramètres sous-tendant les différences observées dans les langues berbères. Les neuf contributions rassemblées dans ce volume abordent ainsi des problèmes précis d'analyse linguistique dans les domaines principaux de la discipline : phonologie, morphologie, syntaxe et sémantique. Elles mettent en évidence de nouvelles généralisations linguistiques, formulées dans un cadre théorique clair, à partir de l'analyse des différents (micro)systèmes que constituent les langues berbères.

6 Ce volume est organisé en deux parties : la première porte sur la documentation et l'analyse de phénomènes et/ou de langues berbères peu connus, la seconde aborde divers phénomènes linguistiques d'un point de vue typologique et comparatif. 


\section{Descriptions et analyses de langues berbères particulières}

7 Dans leur article intitulé Attrition and revival in Awjila Berber, Marijn van Putten \& Lameen Souag s'intéressent au berbère d'Awjila, une langue berbère parlée dans l'est de la Libye. Cette langue, en danger de disparition, possède certains traits uniques dans la famille berbère. Elle est donc d'un intérêt exceptionnel pour les études comparatives et historiques. Pour des raisons politiques, aucune recherche sur le terrain n'a été possible depuis plusieurs décennies. Cependant, plusieurs citoyens d'Awjila ont récemment créé un groupe sur Facebook, Ašal=ənnax (« notre village »), où ils écrivent en awjili. Analysant ce corpus, Marijn van Putten \& Lameen Souag font avancer notre connaissance de cette langue de façon significative. L'étude des traits grammaticaux du corpus révèle une influence claire de l'arabe et un degré d'attrition absent dans les rares données anciennes disponibles. L'article aide par ailleurs à mieux évaluer l'utilité et les limites des médias sociaux comme sources de données linguistiques.

8 La contribution de Maarten Kossmann, The interplay of style, information structure and definiteness: Double indirect objects in Figuig Berber narratives, est consacrée au redoublement datif en berbère de Figuig. Dans cette langue, comme dans d'autres langues berbères, il est possible d'exprimer deux fois l'objet indirect dans le même énoncé, une première fois par une expression lexicale et une seconde fois par un élément pronominal ( redoublement datif »). Cette construction est en variation avec des constructions où l'expression pronominale fait défaut. Comparant un corpus écrit (Benamara 2011) et un corpus oral (recueilli par l'auteur), Maarten Kossmann met en évidence que le redoublement datif est quasiment absent du corpus écrit tandis qu'il est bien attesté dans le corpus oral et montre que, dans le corpus oral, son emploi est clairement corrélé à la structure informative de la phrase.

9 Abdelhak El Hankari explore dans son article, The Copulative Predication in Tarifit Berber, la typologie de la prédication copulative en berbère tarifit. Il identifie trois copules principales : verbale, nominale et locative. Étant donné que tous ces éléments peuvent être employés comme prédicats, l'auteur propose une configuration uniforme qui rend compte de leur dérivation. Cette structure consiste en un niveau lexical inférieur occupé par le prédicat (VP, NP etc.) et une projection fonctionnelle plus haute représentée par le Predicate Phrase (PredP). La tête Pred entre ensuite dans une relation d'accord avec la tête basse située en position de complément, créant une phrase à copule prédicative. Cette analyse unifiée permet de ramener la différence entre les divers prédicats copulatifs au statut catégoriel du prédicat de base occupant la projection lexicale basse.

10 Le dernier article de cette section, celui de José Farrujia de la Rosa, Written on stones. The amazigh colonization of the Canary Islands and the Libyco-Berber and Latino Canarian scripts, est consacré aux inscriptions libyco-berbères des Iles Canaries. L'auteur présente des données archéologiques qui montrent que l'ancienne colonisation des Iles Canaries fut initiée au début du 1er millénaire avant notre ère par des populations Imazighen. Cette colonisation s'accompagna de l'introduction, dans l'archipel des Canaries, d'inscriptions lybico-berbères, parmi d'autres éléments culturels du monde Amazigh d'Afrique du Nord. Dans son article, José Farrujia de la Rosa analyse la 
colonisation ancienne des Iles Canaries à la lumière des inscriptions libyco-berbères, des écritures latines des Iles Canaries et de la culture matérielle indigène.

\section{Typologie et (micro)variation dans la famille berbère}

11 Le premier article de la seconde section du volume, consacrée à la typologie et à la (micro)variation dans la famille berbère, est celui de Mena Lafkioui, Méthodologie de recherche en géolinguistique. L'auteur présente les méthodes principales des études en géolinguistique (outils d'enquêtes de terrain nécessaires à la constitution de corpus de matériaux oraux et visuels qui permettent des analyses géolinguistiques, dont également celles de type comparatif) ainsi que les méthodes cartographiques appropriées pour la représentation de la variation géolinguistique. Ces méthodes sont illustrées à l'aide de l'Atlas linguistique des variétés berbères du Rif (2007) réalisé par l'auteur.

12 Karim Bensoukas présente dans son article, bu-nouns in Tashlhit - An oft-overlooked Complex Morpho-syntactic Corpus, un corpus de noms tachelhit en bu- (bu- exprime généralement le possesseur de ce que désigne le nom interne). Ces noms sont intéressants à deux égards. D'une part, une approche comparatiste avec d'autres parlers amazighs révèle leur complexité inter-dialectale. D'autre part, leur complexité morphosyntaxique représente un défi pour l'Universel 28 de Greenberg, à savoir l'Hypothèse de l'Intégrité Lexicale et l'Absence de Syntagme (No Phrase Constraint). Ces noms n'ont cependant été traités que de façon sporadique dans la littérature. L'article pallie ce manque et met en évidence diverses caractéristiques de ces noms: alternances flexionnelles inhérentes, existence d'une expression périphrastique analogue, pluralisation, modes de possession aliénable et inaliénable, et récurrence des affixes.

13 Le premier but de l'article d'Aïcha Belkadi, Deictic directionality and Space in Berber - A preliminary typological investigation of the semantics of $=d$ and $=n n$, est de présenter une description pan-berbère des sens des clitiques directionnels $=d$ and $=n$, particules qui marquent la trajectoire déictique des verbes de mouvement dans les langues berbères. Le deuxième but est de mettre en évidence le rôle joué par la sémantique verbale dans la distribution de ces clitiques. Adoptant la théorie de lexicalisation de concepts sémantiques de Talmy (2000), l'auteur propose que le type de concept lexicalisé par le verbe modifié par les particules en question gouverne la présence des clitiques et leur sémantique.

14 L'article de Jamal Ouhalla \& Abdelhak El Hankari, Wh-clitic-doubling and whcliticisation: A comparative examination of wh-questions and wh-words in Berber, explore un phénomène selon lequel l'extraction d'arguments datifs (de verbes, noms et prépositions) en berbère donne naissance à deux occurrences de wh. L'une est un mot wh situé en Spec, $C$ et l'autre un clitique wh datif situé en $C$. Un examen précis des données révèle que le mot wh en Spec, $C$ fonctionne comme un opérateur et que le clitique wh datif en $\mathrm{C}$ lui fournit un lien dérivationnel avec la variable dans la position de datif qu'il lie. L'analyse proposée a diverses implications théoriques sur le statut de ce type de cliticisation dans la grammaire, implications explicitées par les auteurs.

Enfin, Sabrina Bendjaballah, Samir Ben Si Saïd \& Martin Haiden, dans le dernier article du volume, intitulé Le syntagme prépositionnel à la périphérie gauche en Taqbaylit, 
examinent les constructions interrogatives portant sur un groupe prépositionnel dans un réseau de 10 points d'enquête en Kabylie. Certaines de ces constructions sont caractérisées par la préfixation de la préposition (P) sur le complémenteur (C). Les auteurs mettent en évidence que si, dans une construction interrogative portant sur un complément prépositionnel, $\mathrm{C}$ est spécifié par un constituant interrogatif, alors $\mathrm{C}$ ne porte pas de $\mathrm{P}$ préfixée. Si $\mathrm{C}$ est spécifié par un constituant non interrogatif ou bien si le spécificateur de C n'est pas réalisé morphologiquement, alors $C$ est préfixé par $\mathrm{P}$. Cet article fait partie d'un projet plus large portant sur la phonologie, la morphologie et la syntaxe des groupes prépositionnels dans différentes variétés du taqbaylit. 